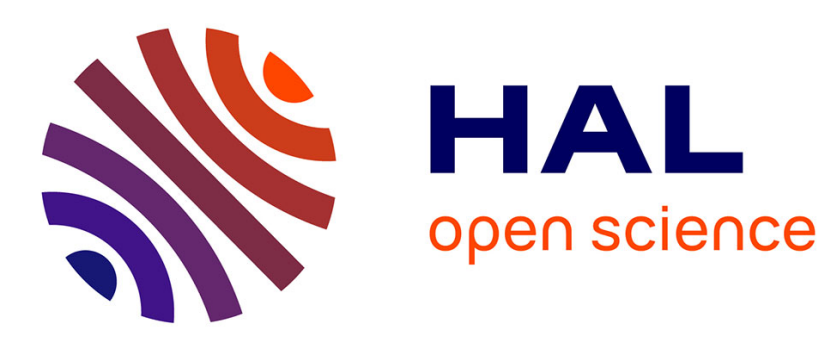

\title{
Multi-Robot Symmetric Formations for Gradient and Hessian Estimation with Application to Source Seeking
}

Lara Briñon Arranz, Alessandro Renzaglia, Luca Schenato

\section{To cite this version:}

Lara Briñon Arranz, Alessandro Renzaglia, Luca Schenato. Multi-Robot Symmetric Formations for Gradient and Hessian Estimation with Application to Source Seeking. IEEE Transactions on Robotics, 2019, 35 (3), pp.782-789. 10.1109/TRO.2019.2895509 . hal-01991153

\section{HAL Id: hal-01991153 \\ https://hal.inria.fr/hal-01991153}

Submitted on 23 Jan 2019

HAL is a multi-disciplinary open access archive for the deposit and dissemination of scientific research documents, whether they are published or not. The documents may come from teaching and research institutions in France or abroad, or from public or private research centers.
L'archive ouverte pluridisciplinaire HAL, est destinée au dépôt et à la diffusion de documents scientifiques de niveau recherche, publiés ou non, émanant des établissements d'enseignement et de recherche français ou étrangers, des laboratoires publics ou privés. 


\title{
Multi-Robot Symmetric Formations for Gradient and Hessian Estimation with Application to Source Seeking
}

\author{
Lara Briñón-Arranz, Alessandro Renzaglia, and Luca Schenato
}

\begin{abstract}
This paper deals with the problem of estimating in a collaborative way the gradient and the Hessian matrix of an unknown signal via noisy measurements collected by a group of robots. We propose symmetric formations with a reduced number of robots for both the two-dimensional (2-D) and the threedimensional (3-D) cases, such that the gradient and Hessian of the signal are estimated at the center of the formation via simple computation on local quantities independently of the orientation of the formation. If only gradient information is required, the proposed formations are suitable for mobile robots that need to move in circular motion. We also provide explicit bounds for the approximation error and for the noise perturbation that can be used to optimally scale the formation radius. Numerical simulations illustrate the performance of the proposed strategy for source seeking against alternative solutions available in the literature and show how Hessian estimation can provide faster convergence even in presence of noisy measurements.
\end{abstract}

Index Terms-Networked Robots, Sensor Networks, Cooperating Robots, Cooperative Estimation, Source seeking.

\section{INTRODUCTION}

$\mathbf{S}$ OURCE localization is a fundamental problem in nature which is relevant to many complex applications, such as environmental monitoring [1], search and rescue operations [2], odor source detection [3] and pollution sensing [4]. The problem of localizing the source of a signal has been approached in two distinct manners: deploying a fixed network of sensors that collect measurements to cooperatively estimate the source location or employing a mobile robot or a robot team equipped with appropriate sensors which is steered toward the source using collected signal measurements while moving (see [5] and the references therein).

The majority of the source seeking strategies proposed in the current literature exploit the measurements collected by a group of mobile robots to estimate properties of the signal such that the model parameters of the scalar field of interest or its gradient. This estimated information can then be used to steer the robots toward the source location, for instance via gradientbased methods. There are several approaches to estimate the gradient of a signal, as extremum seeking techniques [6], least-squares methods [7], [8], consensus-based parametric algorithms [9] and cooperative Kalman filters [10]. However,

L. Briñón-Arranz is with Univ. Grenoble Alpes, CNRS, Grenoble INP, GIPSA-lab, 38000 Grenoble, France (e-mail: lara.brinon-arranz@gipsa-lab.fr)

A. Renzaglia is with Univ. Grenoble Alpes, Inria, 38000 Grenoble, France (e-mail: alessandro.renzaglia@inria.fr)

L. Schenato is with the Department of Information Engineering, University of Padova, 35122 Padova, Italy (e-mail: schenato@dei.unipd.it) all these strategies do not explicitly quantify the quality of the estimation algorithm nor the impact of measurement noise and at best the performance is analyzed only in simulation. Such error analysis is of paramount importance in practical applications to properly design formation shapes or exploration strategies [11]. Such analysis is often avoided in the literature since no specific formation patterns of the robots are enforced, thus resulting in algorithms which are highly nonlinear in the relative distance measures with high communication and computational complexity. Finally, only few works addressed the problem of multi-robot source seeking in 3-D scenarios [12], [13] or proposed strategies to estimate the Hessian of the unknown signal from noisy measurements [7], [14], [15].

Based on the ideas presented in [16], where a strategy to estimate only the gradient of 2-D signals is proposed, this paper aims to overcome the main drawbacks of related works presenting an alternative strategy to cooperatively estimate the gradient and the Hessian matrix of an unknown signal for both 2-D and 3-D scenarios. The major contribution is to show that under some specific symmetric formation patterns, the estimates can be obtained via simple averages that involve only the products of the measurements collected by the robots and their relative position with respect to the formation center. Differently, the aforementioned works typically require the solution of a least-squares problem of the size of the formation size, which then scales quadratically from both a computational and a memory/communication perspective. Besides reduced communication and computational complexity which is appropriate for implementation in small robotic platforms, this approach also provides explicit a priori computation of the approximation error and of the measurements noise error which are shown to be monotonically decreasing and increasing functions of the formation radius, respectively. As so, if the radius is not properly chosen, either the approximation error or the measurement noise can induce poor estimation quality.

To validate the quality of the proposed strategy, we numerically compare it with an alternative least-squares-based solution [8] and we show how Hessian information can be used to drive the center of the multi-robot formation to the location of the source much faster than gradient-based algorithms only if the formation radius is properly chosen, thus stressing the value of theoretical analysis of this work. Additionally, the robustness of the estimations with respect to perturbed robots' positions in the formation is studied through numerical simulations.

The rest of the paper is organized as follows. First, Section II 
states the problem formulation and the paper contributions. Section III exposes the results dealing with the gradient and the Hessian estimation for the 2-D case. Section IV presents the contribution for the 3-D scenario. Section $\mathrm{V}$ characterizes the effect of measurement noise in 3-D scenario and discusses how to optimally determine the formation radius. The performance of the proposed estimation method is analyzed through numerical simulations in Section VI Finally, we present our conclusions and future directions.

\section{PROBLEM FORMULATION}

The main purpose of the source seeking is to steer a group of robots to the source location of a signal distribution. In this setup, each robot represents a mobile sensor or an autonomous vehicle equipped with a sensor that is able to measure the signal strength emitted by the source. In mathematical terms, the signal distribution $\sigma(\mathbf{r})$ is a spatial function representing the scalar field at location $\mathbf{r}$ achieving its maximum in the position $\mathbf{r}^{*}$ where the source is located and smoothly decreasing to zero far from the source. Formally speaking, we will consider the following assumption on this signal, where we denote its gradient and Hessian at a location $\mathbf{r}$ as $\nabla \sigma(\mathbf{r})$ and $\mathbf{H}_{\sigma(\mathbf{r})}$ respectively:

Assumption 1 The function $\sigma: \mathbb{R}^{p} \rightarrow \mathbb{R}^{+}$, where $p=2$ D) or $p=3(3-D)$, is three times continuously differentiable, i.e. $\sigma \in \mathcal{C}^{3}$, and all its partial derivatives up to order three are globally bounded. Moreover, $\nabla \sigma\left(\boldsymbol{r}^{*}\right)=0, \nabla \sigma(\boldsymbol{r}) \neq 0, \forall \boldsymbol{r} \neq$ $\boldsymbol{r}^{*}$, and $\boldsymbol{H}_{\sigma\left(\boldsymbol{r}^{*}\right)}<-a \boldsymbol{I}_{p}$, where $a>0$ and $\boldsymbol{I}_{p} \in \mathbb{R}^{p \times p}$ denotes the identity matrix.

This assumption implies that there exist scalars $L$ and $M$ such that

$$
\begin{gathered}
|\underbrace{\sigma(\mathbf{r})-\sigma(\mathbf{c})-\nabla \sigma(\mathbf{c})^{T}(\mathbf{r}-\mathbf{c})}_{=: \varphi^{\nabla}(\mathbf{r}, \mathbf{c})}| \leq L\|\mathbf{r}-\mathbf{c}\|^{2} \\
|\underbrace{\varphi^{\nabla}(\mathbf{r}, \mathbf{c})-\frac{1}{2}(\mathbf{r}-\mathbf{c})^{T} \mathbf{H}_{\sigma(\mathbf{c})}(\mathbf{r}-\mathbf{c})}_{=: \varphi^{H}(\mathbf{r}, \mathbf{c})}| \leq M\|\mathbf{r}-\mathbf{c}\|^{3}
\end{gathered}
$$

where $\varphi^{\nabla}(\mathbf{r}, \mathbf{c})$ and $\varphi^{H}(\mathbf{r}, \mathbf{c})$ correspond to the first and second order remainders of the Taylor expansion about the point $\mathbf{c}$, respectively.

The knowledge of the gradient and the Hessian can then be used to drive the group of robots towards the source via gradient-ascent (GA) or Newton-Raphson-like ascent (NRA) algorithms, for example by steering the center of robots' formation as follows

$$
\begin{aligned}
\mathbf{c}_{k+1} & =\mathbf{c}_{k}+\varepsilon \nabla \sigma\left(\mathbf{c}_{k}\right), & & \text { GA } \\
\mathbf{c}_{k+1} & =\mathbf{c}_{k}-\varepsilon\left(\left[\mathbf{H}_{\sigma\left(\mathbf{c}_{k}\right)}\right]_{\alpha}\right)^{-1} \nabla \sigma\left(\mathbf{c}_{k}\right), & & \text { NRA }
\end{aligned}
$$

where $\varepsilon>0$ is the step size and the operator $[\cdot]_{\alpha}$ is defined as

$$
[\mathbf{A}]_{\alpha}=\mathbf{A}, \text { if } \mathbf{A} \leq-\alpha \mathbf{I}_{p}, \quad[\mathbf{A}]_{\alpha}=-\mathbf{I}_{p} \text { otherwise }
$$

where $0<\alpha<a$. The previous saturation operator is necessary when the formation center $\mathbf{c}$ is far from the source and the Hessian is likely to be positive semidefinite and therefore drives the robots away from the source. Moreover, when

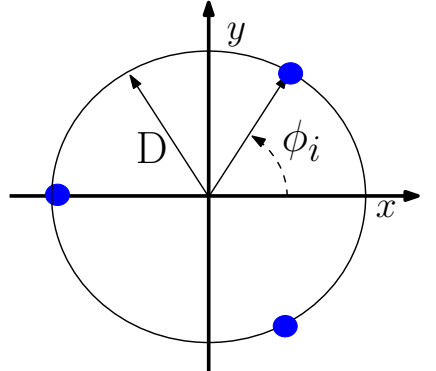

(a)

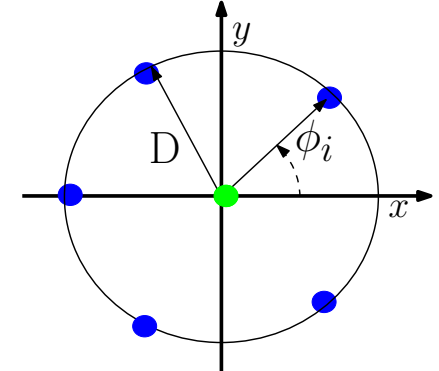

(b)
Fig. 1: Minimal uniformly spaced circular formations for 2-D estimation: (a) formation with $N=3$ robots to estimate the gradient and (b) formation with $N=5$ robots and a green additional robot at the center to estimate the Hessian matrix.

$\mathbf{c} \approx \mathbf{r}^{*}$, by assumption we have that $\mathbf{A}<-a \mathbf{I}_{p}$ and therefore the saturation operator is not active and we recover the standard Newton-Rapshon ascent. A similar approach has been followed in [7]. The core of this manuscript is to provide a procedure to estimate the gradient and the Hessian, referred as $\widehat{\nabla} \sigma(\mathbf{c})$ and $\widehat{\mathbf{H}}_{\sigma(\mathbf{c})}$, based on local measurements by multiple robots in specific symmetric configurations for both 2-D and 3-D settings. Moreover, explicit bounds on the estimation error are provided based on the parameters $L$ and $M$ defined above.

Remark 1 Although we propose a simple kinematic model for source seeking, it can be extended to non-holonomic dynamic models as in [14] and [16], but it is not included here for space limitations. The scope of this work is rather to focus on the gradient and Hessian estimation process in the presence of noisy measurements and to evaluate the benefit of estimating the Hessian to speed up the source seeking process. This direction has only been marginally explored in [7] for 2D scenarios. In 3-D scenarios, Hessian estimation has been proposed in [14] but only to create suitable damping in the gradient-descent process to guarantee stability rather than to speed up source seeking.

\section{2-D ESTIMATION}

\section{A. Robots' formation}

Consider a team of $N$ robots distributed uniformly along a circular formation, as shown in panel (a) of Fig. 1, described by a radius $D$ and a central point $\mathbf{c} \in \mathbb{R}^{2}$, such that

$$
\mathbf{r}_{i}=\mathbf{c}+D \mathbf{R}_{\phi_{i}} \mathbf{e} \quad i=1, \ldots, N
$$

where $\mathbf{r}_{i} \in \mathbb{R}^{2}$ denotes the position of robot $i, \phi_{i}=2 \pi i / N$ is the rotation angle, $\mathbf{R}_{\phi}=\left[\begin{array}{cc}c_{\phi} & -s_{\phi} \\ s_{\phi} & c_{\phi}\end{array}\right]$ denotes the rotation matrix where $c_{\phi}$ and $s_{\phi}$ represent the cosine and sine of angle $\phi$ respectively, and $\mathbf{e}=[1,0]^{T}$. For the sake of simplicity, the robots' dynamics are not considered in this paper. In a previous work of one of the authors, the stabilization problem of a group of vehicles with unicycle-like dynamics to the uniformly distributed circular formation defined by (1) is studied under a cooperative control approach, see [17]. 


\section{B. Gradient estimation}

In the sake of clarity, we briefly recall in this section the results dealing with the gradient estimation of a 2-D signal analyzed in [16].

Theorem 1 Assume that $\sigma(\boldsymbol{r}): \mathbb{R}^{2} \rightarrow \mathbb{R}$ satisfies Assumption 1 and the robots' formation is given by (1). Considering a team of $N \geq 3$ robots uniformly distributed along the circle centered at $\boldsymbol{c}$ and defining

$$
\widehat{\nabla} \sigma(\boldsymbol{c}):=\frac{2}{N D^{2}} \sum_{i=1}^{N} \sigma\left(\boldsymbol{r}_{i}\right)\left(\boldsymbol{r}_{i}-\boldsymbol{c}\right)
$$

then it holds

$$
\|\widehat{\nabla} \sigma(\boldsymbol{c})-\nabla \sigma(\boldsymbol{c})\| \leq L D .
$$

The proof is based on the Taylor series expansion and uses the trigonometric properties of the uniformly distributed circular formation, see [16] for details. This theorem basically states that the estimation error decreases linearly with the formation radius $D$, and therefore can be made arbitrarily small. The following result presents the minimal number of robots required to exactly compute the gradient of a quadratic signal at the center of the circular formation which is relevant since it represents a good approximation of the true signal near the maximum.

Corollary 1 Let $\sigma(\boldsymbol{r}): \mathbb{R}^{2} \rightarrow \mathbb{R}$ be a quadratic function, i.e. $\sigma(\boldsymbol{r})=\boldsymbol{r}^{T} \boldsymbol{S} \boldsymbol{r}+\boldsymbol{p}^{T} \boldsymbol{r}+q$, where $\boldsymbol{S}$ is negative definite, and consider the robots' formation given by (1), then for a team of $N \geq 4$ robots we have $\widehat{\nabla} \sigma(\boldsymbol{c})=\nabla \sigma(\boldsymbol{c})$.

Remark 2 Note that the previous results, as well as the results presented in the sequel, hold for any configuration defined by (1) with $\phi_{i}=\phi_{0}+2 \pi i / N$ where $\phi_{0}$ is an arbitrary rotation angle. As so, the proposed formation is suitable for robots that have to move in circular motion, i.e. $\phi_{0}(t)=\omega_{0} t$, as fixed-wing unmanned aerial vehicles.

\section{Hessian matrix estimation}

This section presents mathematical results dealing with the estimation of the Hessian matrix of a two-dimensional signal. For estimating the Hessian, an additional robot is placed at the formation center as shown in panel (b) of Fig. 1 .

Theorem 2 Assume that $\sigma(\boldsymbol{r}): \mathbb{R}^{2} \rightarrow \mathbb{R}$ satisfies Assumption 11 Let $N$ robots be deployed as in (1) and 1 additional robot placed at the center $\boldsymbol{c}$. If $N \geq 5$ then the quantity:

$$
\boldsymbol{K}_{\sigma(\boldsymbol{c})}:=\frac{16}{N D^{4}} \sum_{i=1}^{N}\left(\sigma\left(\boldsymbol{r}_{i}\right)-\sigma(\boldsymbol{c})\right)\left(\boldsymbol{r}_{i}-\boldsymbol{c}\right)\left(\boldsymbol{r}_{i}-\boldsymbol{c}\right)^{T}
$$

satisfies

$$
\left\|\boldsymbol{K}_{\sigma(\boldsymbol{c})}-\left(3 \boldsymbol{H}_{\sigma(\boldsymbol{c})}+\boldsymbol{R}_{\frac{\pi}{2}} \boldsymbol{H}_{\sigma(\boldsymbol{c})} \boldsymbol{R}_{\frac{\pi}{2}}^{T}\right)\right\|_{F} \leq 16 M D
$$

where $\|\cdot\|_{F}$ indicates the Frobenius norm.

Proof 1 Let us preliminary define the notation $\nabla \sigma(\boldsymbol{c})=$ $\left[\nabla \sigma_{x}(\boldsymbol{c}) \nabla \sigma_{y}(\boldsymbol{c})\right]^{T}$ and $\boldsymbol{H}_{\sigma(\boldsymbol{c})}=\left[\begin{array}{cc}H_{11} & \star \\ H_{12} & H_{22}\end{array}\right] \in \mathbb{R}^{2 \times 2}$ where
* stands for the symmetric terms of the matrix. Using the first-order Taylor expansion of each measurement $\sigma\left(\boldsymbol{r}_{i}\right)$ about the point $\boldsymbol{c}$ and recalling that $\left\|\widetilde{\boldsymbol{r}}_{i}\right\|=D$ where $\widetilde{\boldsymbol{r}}_{i}:=\boldsymbol{r}_{i}-\boldsymbol{c}$, then the following equation holds for all $i=1, \ldots, N$ :

$$
\sigma\left(\boldsymbol{r}_{i}\right)-\sigma(\boldsymbol{c})=\nabla \sigma(\boldsymbol{c})^{T} \widetilde{\boldsymbol{r}}_{i}+\frac{1}{2} \widetilde{\boldsymbol{r}}_{i}^{T} \boldsymbol{H}_{\sigma(\boldsymbol{c})} \widetilde{\boldsymbol{r}}_{i}+\varphi^{H}\left(\boldsymbol{r}_{i}, \boldsymbol{c}\right)
$$

where $\varphi^{H}\left(\boldsymbol{r}_{i}, \boldsymbol{c}\right)$ denotes the remainder of the Taylor expansion. Pre-multiplying the previous equation by $16 \widetilde{\boldsymbol{r}}_{i} /\left(N D^{4}\right)$ and post-multiplying it by $\widetilde{\boldsymbol{r}}_{i}^{T}$, and summing over $i=1, \ldots, N$, we obtain

$$
\begin{aligned}
& \frac{16}{N D^{4}} \sum_{i=1}^{N}\left(\sigma\left(\boldsymbol{r}_{i}\right)-\sigma(\boldsymbol{c})\right) \widetilde{\boldsymbol{r}}_{i} \widetilde{\boldsymbol{r}}_{i}^{T}=\frac{16}{N D^{4}} \sum_{i=1}^{N} \widetilde{\boldsymbol{r}}_{i} \nabla \sigma(\boldsymbol{c})^{T} \widetilde{\boldsymbol{r}}_{i} \widetilde{\boldsymbol{r}}_{i}^{T} \\
& +\frac{8}{N D^{4}} \sum_{i=1}^{N} \widetilde{\boldsymbol{r}}_{i} \widetilde{\boldsymbol{r}}_{i}^{T} \boldsymbol{H}_{\sigma(\boldsymbol{c})} \widetilde{\boldsymbol{r}}_{i} \widetilde{\boldsymbol{r}}_{i}^{T}+\frac{16}{N D^{4}} \sum_{i=1}^{N} \widetilde{\boldsymbol{r}}_{i} \varphi^{H}\left(\boldsymbol{r}_{i}, \boldsymbol{c}\right) \widetilde{\boldsymbol{r}}_{i}^{T} .
\end{aligned}
$$

We start by analyzing the first summatory of the right-side equation:

$$
\begin{aligned}
& \sum_{i=1}^{N} \widetilde{\boldsymbol{r}}_{i} \nabla \sigma(\boldsymbol{c})^{T} \widetilde{\boldsymbol{r}}_{i} \widetilde{\boldsymbol{r}}_{i}^{T}=D^{3} \sum_{i=1}^{N} \boldsymbol{R}_{\phi_{i}} \boldsymbol{e} \nabla \sigma(\boldsymbol{c})^{T} \boldsymbol{R}_{\phi_{i}} \boldsymbol{e} \boldsymbol{e}^{T} \boldsymbol{R}_{\phi_{i}}^{T} \\
& =D^{3} \sum_{i=1}^{N}\left(\nabla \sigma_{x}(\boldsymbol{c})\left[\begin{array}{cc}
c_{\phi_{i}}^{3} & c_{\phi_{i}}^{2} s_{\phi_{\phi^{2}}} \\
c_{\phi_{i}}^{2} s_{\phi_{i}} & c_{\phi_{i}} s_{\phi_{i}}
\end{array}\right]+\nabla \sigma_{y}(\boldsymbol{c})\left[\begin{array}{cc}
c_{\phi_{i}}^{2} s_{\phi_{i}} & c_{\phi_{i}} s_{\phi_{i}}^{2} \\
c_{\phi_{i}} s_{\phi_{i}}^{2} & s_{\phi_{i}}^{3}
\end{array}\right]\right)=\mathbf{0}
\end{aligned}
$$

where we used trigonometric properties that allow to write the powers $c_{\phi}^{m}, c_{\phi}^{m}$ as linear combinations of $s_{\phi}, c_{\phi}, \ldots, s_{m \phi}, c_{m \phi}$ and the fact $\sum_{i=1}^{N} \cos (m 2 \pi i / N)=\sum_{i=1}^{N} \sin (m 2 \pi i / N)=0$ for $N>m$ (see Appendix for details). Since the first element includes terms with $m=3$ we need $N \geq 4$ to satisfy the previous property. Let us now consider the second term of the right-side equation containing the Hessian matrix:

$$
\begin{aligned}
& \frac{8}{N D^{4}} \sum_{i=1}^{N} \widetilde{\boldsymbol{r}}_{i} \widetilde{\boldsymbol{r}}_{i}^{T} \boldsymbol{H}_{\sigma(\boldsymbol{c})} \widetilde{\boldsymbol{r}}_{i} \widetilde{\boldsymbol{r}}_{i}^{T}= \\
& \frac{8}{N} \sum_{i=1}^{N} \boldsymbol{R}_{\phi_{i}} \boldsymbol{e} \boldsymbol{e}^{T} \boldsymbol{R}_{\phi_{i}}^{T} \boldsymbol{H}_{\sigma(\boldsymbol{c})} \boldsymbol{R}_{\phi_{i}} \boldsymbol{e} e^{T} \boldsymbol{R}_{\phi_{i}}^{T}=\frac{8}{N} \sum_{i=1}^{N}\left[\begin{array}{ll}
b_{11} & b_{12} \\
b_{12} & b_{22}
\end{array}\right]
\end{aligned}
$$

where

$$
\begin{aligned}
& b_{11}=H_{11} c_{\phi_{i}}^{4}+2 H_{12} c_{\phi_{i}}^{3} s_{\phi_{i}}+H_{22} c_{\phi_{i}}^{2} s_{\phi_{i}}^{2}, \\
& b_{12}=H_{11} c_{\phi_{i}}^{3} s_{\phi_{i}}+2 H_{12} c_{\phi_{i}}^{2} s_{\phi_{i}}^{2}+H_{22} c_{\phi_{i}} s_{\phi_{i}}^{3}, \\
& b_{22}=H_{11} c_{\phi_{i}}^{2} s_{\phi_{i}}^{2}+2 H_{12} c_{\phi_{i}} s_{\phi_{i}}^{3}+H_{22} s_{\phi_{i}}^{4} .
\end{aligned}
$$

Applying trigonometric properties, and observing that it includes fourth-power trigonometric terms, similarly as before we need to have $N \geq 5$ for the following equation to hold:

$$
\begin{aligned}
\frac{8}{N} \sum_{i=1}^{N}\left[\begin{array}{ll}
b_{11} & b_{12} \\
b_{12} & b_{22}
\end{array}\right] & =8\left[\begin{array}{cc}
\frac{3}{8} H_{11}+\frac{1}{8} H_{22} & \frac{1}{4} H_{12} \\
\frac{1}{4} H_{12} & \frac{1}{8} H_{11}+\frac{3}{8} H_{22}
\end{array}\right] \\
& =3 \boldsymbol{H}_{\sigma(\boldsymbol{c})}+\boldsymbol{R}_{\frac{\pi}{2}} \boldsymbol{H}_{\sigma(\boldsymbol{c})} \boldsymbol{R}_{\frac{\pi}{2}}^{T} .
\end{aligned}
$$

We define the approximation error as follows

$$
\boldsymbol{\Psi}^{H}(D, \boldsymbol{c})=\frac{16}{N D^{4}} \sum_{i=1}^{N}\left(\boldsymbol{r}_{i}-\boldsymbol{c}\right) \varphi^{H}\left(\boldsymbol{r}_{i}, \boldsymbol{c}\right)\left(\boldsymbol{r}_{i}-\boldsymbol{c}\right)^{T}
$$


and by Assumption 1 this error can be bounded as

$$
\left\|\Psi^{H}(D, \boldsymbol{c})\right\|_{F} \leq \frac{16}{N D^{4}} \sum_{i=1}^{N}\left|\varphi^{H}\left(\boldsymbol{r}_{i}, \boldsymbol{c}\right)\right|\left\|\boldsymbol{r}_{i}-\boldsymbol{c}\right\|^{2} \leq 16 M D .
$$

Note that once $\mathbf{K}_{\sigma(\mathbf{c})}$ is computed, an approximation for the Hessian is obtained by the unique solution of the linear system

$$
3 \widehat{\mathbf{H}}_{\sigma(\mathbf{c})}+\mathbf{R}_{\frac{\pi}{2}} \widehat{\mathbf{H}}_{\sigma(\mathbf{c})} \mathbf{R}_{\frac{\pi}{2}}^{T}=\mathbf{K}_{\sigma(\mathbf{c})}
$$

which can be easily computed.

The case of quadratic signals is also analyzed and the minimal number of robots required to exactly compute the Hessian matrix at the center of the circular formation is presented in the sequel:

Corollary 2 Let $\sigma(\boldsymbol{r}): \mathbb{R}^{2} \rightarrow \mathbb{R}$ be a quadratic function, i.e. $\sigma(\boldsymbol{r})=\boldsymbol{r}^{T} \boldsymbol{S} \boldsymbol{r}+\boldsymbol{p}^{T} \boldsymbol{r}+q$, where $\boldsymbol{S}$ is negative definite, and let $N$ robots be deployed as in (1) and 1 additional robot placed at the center $\boldsymbol{c}$. Then for $N \geq 5$, the matrix $\boldsymbol{K}_{\sigma(\boldsymbol{c})}$ defined in (4) satisfies $\boldsymbol{K}_{\sigma(\boldsymbol{c})}=3 \boldsymbol{H}_{\sigma(\boldsymbol{c})}+\boldsymbol{R}_{\frac{\pi}{2}} \boldsymbol{H}_{\sigma(c)} \boldsymbol{R}_{\frac{\pi}{2}}^{T}$ and therefore $\widehat{\boldsymbol{H}}_{\sigma(\boldsymbol{c})}=\boldsymbol{H}_{\sigma(\boldsymbol{c})}$.

The previous result follows directly from Theorem 2 since in the case of quadratic functions the Taylor's remainders are zero, i.e. $\varphi^{H}\left(\mathbf{r}_{i}, \mathbf{c}\right)=0, \forall i=1, \ldots, N$.

Remark 3 The proposed strategy resembles a sampled version of the Poisson integral for harmonic functions if $\sigma(\boldsymbol{c})$ is replaced with $\frac{1}{N} \sum_{i=1}^{N} \sigma\left(\boldsymbol{r}_{i}\right)$ in (4) as proposed in [14]. However, such procedure is suitable only for harmonic signals $\sigma(\cdot)$ which are solutions of diffusion equations at steady state, while our proposed strategy can be applied to any signal which is twice differentiable. For example a quadratic function, which is a relevant approximation of the signal near its maximum, is not a harmonic function.

\section{3-D ESTIMATION}

\section{A. Robots' formation}

Consider a team of $N=2 n, n \in \mathbb{N}$ robots forming a symmetric configuration composed of two parallel circular formations whose centers are aligned with the $z$-axis as shown in panel (a) of Fig. 2 The center point $\mathbf{c} \in \mathbb{R}^{3}$ is located between the two circles at distance $D \sin \theta_{F}$ from each one. One half of the robots is uniformly distributed in the upper circular formation, the other half is uniformly distributed in the lower circular formation and thus, their relative vectors with respect to the center point $\mathbf{c}$ are also evenly spaced, see Fig. 2 . The robots' positions in such configuration are expressed in spherical coordinates as follows:

$$
\begin{aligned}
\mathbf{r}_{i} & =\mathbf{c}+D\left[s_{\theta_{i}} c_{\phi_{i}}, s_{\theta_{i}} s_{\phi_{i}}, c_{\theta_{i}}\right]^{T} \\
\theta_{i} & =\left\{\begin{array}{ll}
\theta_{F}, & \text { if } i=2 m-1 \\
\pi-\theta_{F}, & \text { if } i=2 m,
\end{array} \quad m=1, \ldots, n\right.
\end{aligned}
$$

where $\mathbf{r}_{i} \in \mathbb{R}^{3}$ denotes the position of robot $i, \phi_{i}=2 \pi i / N$ is the azimuthal angle, $D$ the radial distance to the center $\mathbf{c}, \theta_{i}$ is the polar angle and $\theta_{F}$ is defined such that $s_{\theta_{F}}=\sqrt{2 / 3}$.

Note that this symmetric formation has two interesting and convenient properties. First of all, it is easy to see that all the

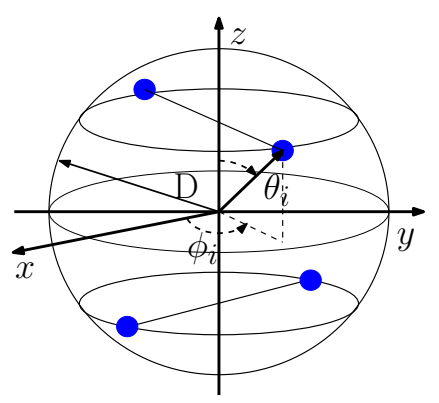

(a)

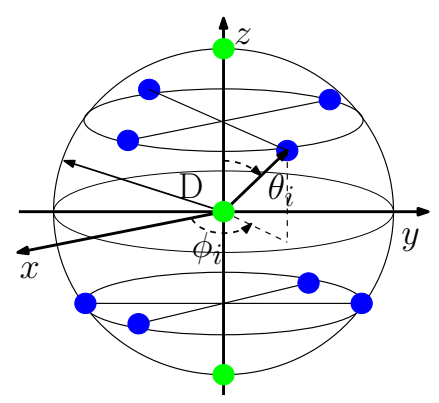

(b)
Fig. 2: Minimal symmetric cylindrical formations for 3-D estimation: (a) formation with $N=4$ robots to estimate the gradient and (b) formation with $N=8$ robots and three additional robots in green to estimate the Hessian matrix.

robots are placed at the same distance from the center, i.e., $\left\|\mathbf{r}_{i}-\mathbf{c}\right\|=D, \forall i$. Moreover, the sum of the relative position vectors of all robots with respect to the center is equal to zero for $N=2 n, n \geq 2$, as proven in the sequel:

$$
\sum_{i=1}^{N}\left(\mathbf{r}_{i}-\mathbf{c}\right)=D\left[\begin{array}{c}
s_{\theta_{F}} \sum_{i=1}^{N} c_{\phi_{i}} \\
s_{\theta_{F}} \sum_{i=1}^{N} s_{\phi_{i}} \\
\sum_{i=2 m-1} c_{\theta_{F}}-\sum_{i=2 m} c_{\theta_{F}}
\end{array}\right]=\mathbf{0}
$$

since $\sum_{i=1}^{N} \cos (2 \pi i / N)=\sum_{i=1}^{N} \sin (2 \pi i / N)=0$.

\section{B. Gradient estimation}

Consider the symmetric formation of robots given by (6) collecting measurements of a 3-D signal distribution $\sigma(\mathbf{r})$. The following theorem is proposed:

Theorem 3 Assume that $\sigma(\boldsymbol{r}): \mathbb{R}^{3} \rightarrow \mathbb{R}$ satisfies Assumption 1 and the robots' formation is given by (6). Considering a team of $N=2 n$ robots with $n \geq 2$ and defining

$$
\widehat{\nabla} \sigma(\boldsymbol{c}):=\frac{3}{N D^{2}} \sum_{i=1}^{N} \sigma\left(\boldsymbol{r}_{i}\right)\left(\boldsymbol{r}_{i}-\boldsymbol{c}\right)
$$

then it holds

$$
\|\widehat{\nabla} \sigma(\boldsymbol{c})-\nabla \sigma(\boldsymbol{c})\| \leq 3 L D .
$$

Proof 2 Using the first-order Taylor expansion of each measurement $\sigma\left(\boldsymbol{r}_{i}\right)$ about the point $\boldsymbol{c}$ and recalling that $\left\|\widetilde{\boldsymbol{r}}_{i}\right\|=D$ where $\widetilde{\boldsymbol{r}}_{i}:=\boldsymbol{r}_{i}-\boldsymbol{c}$, then the following equation holds for all $i=1, \ldots, N$ :

$$
\sigma\left(\boldsymbol{r}_{i}\right)-\sigma(\boldsymbol{c})=\nabla \sigma(\boldsymbol{c})^{T} \widetilde{\boldsymbol{r}}_{i}+\varphi^{\nabla}\left(\boldsymbol{r}_{i}, \boldsymbol{c}\right),
$$

where $\varphi^{\nabla}\left(\boldsymbol{r}_{i}, \boldsymbol{c}\right)$ denotes the remainder of the Taylor expansion. Multiplying the previous equation by $3 \widetilde{\boldsymbol{r}}_{i} /\left(N D^{2}\right)$ and summing over $i=1, \ldots, N$, we obtain

$$
\begin{aligned}
& \frac{3}{N D^{2}} \sum_{i=1}^{N} \sigma\left(\boldsymbol{r}_{i}\right) \widetilde{\boldsymbol{r}}_{i}-\frac{3 \sigma(\boldsymbol{c})}{N D^{2}} \sum_{i=1}^{N} \widetilde{\boldsymbol{r}}_{i}= \\
& \frac{3}{N D^{2}} \sum_{i=1}^{N} \nabla \sigma(\boldsymbol{c})^{T} \widetilde{\boldsymbol{r}}_{i} \widetilde{\boldsymbol{r}}_{i}+\frac{3}{N D^{2}} \sum_{i=1}^{N} \varphi^{\nabla}\left(\boldsymbol{r}_{i}, \boldsymbol{c}\right) \widetilde{\boldsymbol{r}}_{i} .
\end{aligned}
$$


Due to the symmetric properties of the formation, we have that $\sum_{i=1}^{N} \widetilde{\boldsymbol{r}}_{i}=0$ and thus

$$
\widehat{\nabla} \sigma(\boldsymbol{c})=\frac{3 \nabla \sigma(\boldsymbol{c})}{N D^{2}} \sum_{i=1}^{N} \widetilde{\boldsymbol{r}}_{i} \widetilde{\boldsymbol{r}}_{i}^{T}+\boldsymbol{\Psi}^{\nabla}(D, \boldsymbol{c}),
$$

where $\Psi^{\nabla}(D, \boldsymbol{c})=\frac{3}{N D^{2}} \sum_{i=1}^{N} \varphi^{\nabla}\left(\boldsymbol{r}_{i}, \boldsymbol{c}\right) \widetilde{\boldsymbol{r}}_{i}$. We analyze the second term of the previous equation using to to express the position of the robots $\boldsymbol{r}_{i}$ to obtain

$$
\sum_{i=1}^{N} \widetilde{\boldsymbol{r}}_{i} \widetilde{\boldsymbol{r}}_{i}^{T}=D^{2} \sum_{i=1}^{N}\left[\begin{array}{ccc}
s_{\theta_{F}}^{2} c_{\phi_{i}}^{2} & \star & \star \\
s_{\theta_{F}}^{2} c_{\phi_{i}} s_{\phi_{i}} & s_{\theta_{F}}^{2} s_{\phi_{i}}^{2} & \star \\
s_{\theta_{F}} c_{\phi_{i}} c_{\theta_{i}} & s_{\theta_{F}} s_{\phi_{i}} c_{\theta_{i}} & c_{\theta_{i}}^{2}
\end{array}\right] .
$$

The elements depending on the polar angle $\theta_{i}$ must be decomposed to consider the robots with even index $i=2 m$ and the rest with $i=2 m-1, m=1, \ldots, n$, then for $n \geq 2$ we have

$$
\sum_{i=1}^{N} c_{\phi_{i}} c_{\theta_{i}}=c_{\theta_{F}}\left(\sum_{i=2 m-1}^{n} c_{\phi_{i}}-\sum_{i=2 m}^{n} c_{\phi_{i}}\right)=0
$$

and the same results holds for the sine. Considering the properties of the proposed formation (6) for which $s_{\theta_{F}}^{2}=2 / 3$, and applying trigonometric properties, the following equality holds:

$$
\sum_{i=1}^{N} \widetilde{\boldsymbol{r}}_{i} \widetilde{\boldsymbol{r}}_{i}^{T}=D^{2}\left[\begin{array}{ccc}
\frac{N}{2} s_{\theta_{F}}^{2} & \star & \star \\
0 & \frac{N}{2} s_{\theta_{F}}^{2} & \star \\
0 & 0 & N c_{\theta_{F}}^{2}
\end{array}\right]=\frac{N D^{2}}{3} \boldsymbol{I}_{3},
$$

consequently $\widehat{\nabla} \sigma(\boldsymbol{c})=\nabla \sigma(\boldsymbol{c})+\boldsymbol{\Psi}^{\nabla}(D, \boldsymbol{c})$. According to Assumption 1 the term $\Psi^{\nabla}(D, c)$ satisfies the inequality

$$
\left\|\Psi^{\nabla}(D, \boldsymbol{c})\right\| \leq \frac{3}{N D^{2}} \sum_{i=1}^{N}\left|\varphi^{\nabla}\left(\boldsymbol{r}_{i}, \boldsymbol{c}\right)\right|\left\|\boldsymbol{r}_{i}-\boldsymbol{c}\right\| \leq 3 L D,
$$

which concludes the proof.

Following the same ideas of Theorem 3, it can be proven that the gradient of a 3-D quadratic signal can be exactly computed by the symmetric configuration defined by (6):

Corollary 3 Let $\sigma(\boldsymbol{r}): \mathbb{R}^{3} \rightarrow \mathbb{R}$ be a quadratic function, i.e. $\sigma(\boldsymbol{r})=\boldsymbol{r}^{T} \boldsymbol{S} \boldsymbol{r}+\boldsymbol{p}^{T} \boldsymbol{r}+q$, where $\boldsymbol{S}$ is negative definite, and consider the robots' formation given by (6), then for a team of $N=2 n$ robots with $n \geq 3$ we have $\widehat{\nabla} \sigma(\boldsymbol{c})=\nabla \sigma(\boldsymbol{c})$.

\section{Hessian matrix estimation}

Consider the symmetric formation of robots given by (6) taking measurements of a signal distribution $\sigma(\mathbf{r})$. In order to estimate the Hessian matrix of the signal $\sigma(\mathbf{r})$, three additional robots are required, two placed along the $z$-axis of the previous symmetric formation, such that the robots positions are given by (6) with $\theta_{N+1}=0$ and $\theta_{N+2}=\pi$ and another one placed at the center $\mathbf{c}$ of the formation, as shown in panel (b) of Fig. 2. The following theorem is proposed:

Theorem 4 Assume that $\sigma(\boldsymbol{r}): \mathbb{R}^{3} \rightarrow \mathbb{R}$ satisfies Assumption 1. Let $N=2 n$ robots deployed as in (6), two additional robots are defined by (6) with $\theta_{N+1}=0$ and $\theta_{N+2}=\pi$ respectively, and the robot $N+3$ is placed at the center $c$ of the formation. If $n \geq 4$ then the quantity

$$
\boldsymbol{K}_{\sigma(\boldsymbol{c})}:=\frac{18}{N D^{4}} \sum_{i=1}^{N+2}\left(\sigma\left(\boldsymbol{r}_{i}\right)-\sigma(\boldsymbol{c})\right)\left(\boldsymbol{r}_{i}-\boldsymbol{c}\right)\left(\boldsymbol{r}_{i}-\boldsymbol{c}\right)^{T}
$$

satisfies

$$
\|\boldsymbol{K}_{\sigma(\boldsymbol{c})}-\underbrace{\left[\begin{array}{ccc}
d_{1} & \star & \star \\
H_{12} & d_{2} & \star \\
2 H_{13} & 2 H_{23} & d_{3}
\end{array}\right]}_{=: \mathcal{L}\left(\boldsymbol{H}_{\sigma(\boldsymbol{c})}\right)}\|_{F} \leq \frac{18(N+2) D M}{N}
$$

with

$$
\begin{aligned}
& d_{1}=3 H_{11} / 2+H_{22} / 2+H_{33} \\
& d_{2}=H_{11} / 2+3 H_{22} / 2+H_{33} \\
& d_{3}=H_{11}+H_{22}+H_{33}+18 H_{33} / N
\end{aligned}
$$

where the Hessian matrix at the center of the formation is denoted by $\boldsymbol{H}_{\sigma(\boldsymbol{c})}=\left[\begin{array}{lll}H_{11} & \star & \star \\ H_{12} & H_{22} & \star \\ H_{13} & H_{23} & H_{33}\end{array}\right]$.

Proof 3 Using the first-order Taylor expansion of each measurement $\sigma\left(\boldsymbol{r}_{i}\right)$ about the point $\boldsymbol{c}$ and recalling that $\left\|\widetilde{\boldsymbol{r}}_{i}\right\|=D$, where $\widetilde{\boldsymbol{r}}_{i}:=\boldsymbol{r}_{i}-\boldsymbol{c}$ then the following equation holds for all $i=1, \ldots, N+2$ :

$$
\sigma\left(\boldsymbol{r}_{i}\right)-\sigma(\boldsymbol{c})=\nabla \sigma(\boldsymbol{c})^{T} \widetilde{\boldsymbol{r}}_{i}+\frac{1}{2} \widetilde{\boldsymbol{r}}_{i}^{T} \boldsymbol{H}_{\sigma(\boldsymbol{c})} \widetilde{\boldsymbol{r}}_{i}+\varphi^{H}\left(\boldsymbol{r}_{i}, \boldsymbol{c}\right),
$$

where $\varphi^{H}\left(\boldsymbol{r}_{i}, \boldsymbol{c}\right)$ denotes the remainder of the Taylor expansion. Pre-multiplying the previous equation by $18 \widetilde{\boldsymbol{r}}_{i} /\left(N D^{4}\right)$ and post-multiplying by $\widetilde{\boldsymbol{r}}_{i}^{T}$, and then summing over $i=$ $1, \ldots, N+2$, we obtain

$$
\begin{aligned}
& \boldsymbol{K}_{\sigma(\boldsymbol{c})}=\frac{18}{N D^{4}} \sum_{i=1}^{N+2} \widetilde{\boldsymbol{r}}_{i} \nabla \sigma(\boldsymbol{c})^{T} \widetilde{\boldsymbol{r}}_{i} \widetilde{\boldsymbol{r}}_{i}^{T}+ \\
& +\frac{9}{N D^{4}} \sum_{i=1}^{N+2} \widetilde{\boldsymbol{r}}_{i} \widetilde{\boldsymbol{r}}_{i}^{T} \boldsymbol{H}_{\sigma(\boldsymbol{c})} \widetilde{\boldsymbol{r}}_{i} \widetilde{\boldsymbol{r}}_{i}^{T}+\frac{18}{N D^{4}} \sum_{i=1}^{N+2} \widetilde{\boldsymbol{r}}_{i} \varphi^{H}\left(\boldsymbol{r}_{i}, \boldsymbol{c}\right) \widetilde{\boldsymbol{r}}_{i}^{T}
\end{aligned}
$$

To analyze this equation, we decompose the summation in two parts: first, consider the $N$ robots of the cylindrical formation and second, the two additional ones. Following the same steps that in previous cases, applying trigonometric properties and considering robots with even and odd index, we can show that the first term of the right-side of the previous equation is equal to zero for $n \geq 4$ and the second term is equal to

$$
\left[\begin{array}{ccc}
\frac{3}{2} H_{11}+\frac{1}{2} H_{22}+H_{33} & \star & \star \\
H_{12} & \frac{1}{2} H_{11}+\frac{3}{2} H_{22}+H_{33} & \star \\
2 H_{13} & 2 H_{23} & H_{11}+H_{22}+H_{33}+\frac{18}{N} H_{33}
\end{array}\right] \text {. }
$$

Additional details on the mathematical derivations can be found in [18]. The error term defined by

$$
\boldsymbol{\Psi}^{H}(D, \boldsymbol{c})=\frac{18}{N D^{4}} \sum_{i=1}^{N+2} \widetilde{\boldsymbol{r}_{i}} \varphi^{H}\left(\boldsymbol{r}_{i}, \boldsymbol{c}\right) \widetilde{\boldsymbol{r}}_{i}^{T}
$$

satisfies

$$
\left\|\Psi^{H}(D, \boldsymbol{c})\right\|_{F} \leq \frac{18}{N D^{4}} \sum_{i=1}^{N+2} \mid \varphi^{H}\left(\boldsymbol{r}_{i}, \boldsymbol{c}\right)\left\|\widetilde{\boldsymbol{r}}_{i}\right\|^{2} \leq \frac{18(N+2) D M}{N} .
$$


Note that once $\mathbf{K}_{\sigma(\mathbf{c})}$ is computed, an estimate $\widehat{\mathbf{H}}_{\sigma(\mathbf{c})}$ for the true Hessian $\mathbf{H}_{\sigma(\mathbf{c})}$ is given by the unique solution of the linear system

$$
\mathcal{L}\left(\widehat{\mathbf{H}}_{\sigma(\mathbf{c})}\right)=\mathbf{K}_{\sigma(\mathbf{c})}
$$

which can be obtained by inverting the operator $\mathcal{L}$.

Similarly to the 2-D setting, the case of a quadratic signal is also analyzed in the sequel:

Corollary 4 Let $\sigma(\boldsymbol{r}): \mathbb{R}^{3} \rightarrow \mathbb{R}$ be a quadratic function, i.e. $\sigma(\boldsymbol{r})=\boldsymbol{r}^{T} \boldsymbol{S} \boldsymbol{r}+\boldsymbol{p}^{T} \boldsymbol{r}+q$, where $\boldsymbol{S}$ is negative definite. Considering $N=2 n$ robots deployed as in (6), two additional robots are defined by (6) with $\theta_{N+1}=0$ and $\theta_{N+2}=\pi$ respectively, and the robot $N+3$ is placed at the center $\boldsymbol{c}$ of the formation. Then for $n \geq 4$ the matrix $\boldsymbol{K}_{\sigma(\boldsymbol{c})}$ defined in (10) satisfies $\boldsymbol{K}_{\sigma(\boldsymbol{c})}=\mathcal{L}\left(\boldsymbol{H}_{\sigma(\boldsymbol{c})}\right)$ and therefore $\widehat{\boldsymbol{H}}_{\sigma(\boldsymbol{c})}=\boldsymbol{H}_{\sigma(\boldsymbol{c})}$.

Remark 4 Both Corollaries 3 and 4 present the minimal number of robots required to exactly compute the gradient and the Hessian matrix of quadratic signals at the center of the 3-D symmetric formation, respectively. Details of the proofs can be found in [18].

\section{NOISE AND APPROXIMATION ERROR ANALYSIS}

In this section we address the problem of noisy measurements and their effect on the gradient and Hessian estimation, and we compare it with the approximation errors of equations (8) and (11). We limit the analysis to the 3-D scenario, being the 2-D scenario very similar. We assume that the measurements are corrupted by Gaussian zero-mean white noise which models possible small scale spatial variations due to turbulence or local perturbation:

$$
y\left(\mathbf{r}_{i}\right)=\sigma\left(\mathbf{r}_{i}\right)+v_{i}\left(\mathbf{r}_{i}\right), \quad v_{i}\left(\mathbf{r}_{i}\right) \sim \mathcal{N}\left(0, v^{2}\right)
$$

where $v^{2}$ represents the variance of the noise. We further assume that the noise is independent in each robot measurement, i.e. $\mathbb{E}\left[v_{i}\left(\mathbf{r}_{i}\right) v_{j}\left(\mathbf{r}_{j}\right)\right]=0, i \neq j$ which is a realistic assumption if $\left\|\mathbf{r}_{i}-\mathbf{r}_{j}\right\|>D_{\min }$ where $D_{\min }$ represents the spatial correlation distance of the disturbance. We start by considering the effect on the gradient estimation. Since the noise is additive in the measurement, the gradient estimate defined in (7) becomes

$$
\widehat{\nabla} \sigma(\mathbf{c}):=\frac{3}{N D^{2}} \sum_{i=1}^{N} \sigma\left(\mathbf{r}_{i}\right)\left(\mathbf{r}_{i}-\mathbf{c}\right)+\underbrace{\frac{3}{N D^{2}} \sum_{i=1}^{N} v_{i}\left(\mathbf{r}_{i}-\mathbf{c}\right)}_{\Psi_{v}^{\nabla}(D, \mathbf{c})} .
$$

The term $\boldsymbol{\Psi}_{v}^{\nabla}(D, \mathbf{c})$ represents the effect of noise. It has clearly zero mean, i.e. $\mathbb{E}\left[\mathbf{\Psi}_{v}^{\nabla}(D, \mathbf{c})\right]=0$ and its expected standard deviation is given by:

$$
\begin{aligned}
\mathrm{s}_{\nabla}(D) & :=\sqrt{\mathbb{E}\left[\left\|\Psi_{v}^{\nabla}(D, \mathbf{c})\right\|^{2}\right]}=\frac{3}{N D^{2}}\left(\mathbb{E}\left[\sum_{i=1}^{N} \sum_{j=1}^{N} v_{i} v_{j} \widetilde{\mathbf{r}}_{i}^{T} \widetilde{\mathbf{r}}_{j}\right]\right)^{\frac{1}{2}} \\
& =\frac{3 v}{N D^{2}}\left(\sum_{i=1}^{N} \widetilde{\mathbf{r}}_{i}^{T} \widetilde{\mathbf{r}}_{i}\right)^{\frac{1}{2}}=\frac{3 v}{N^{\frac{1}{2}} D},
\end{aligned}
$$

where we used the results from the proof of Theorem 3 . Note that this term is a monotonically decreasing function of the formation radius and the number of robots. This term has to be compared with the approximation error provided in (8) which is monotonically increasing with the formation radius. This observation shows a clear trade-off when deciding the formation radius in order to balance the effect of approximation error and the noise error. As a rule-of-thumb we can try to design the formation radius $D_{\nabla}^{*}$ to exactly balance these two errors (see [18] for more details):

$\mathrm{s}_{\nabla}\left(D_{\nabla}^{*}\right)=3 L D_{\nabla}^{*} \Longrightarrow D_{\nabla}^{*}=\frac{v^{\frac{1}{2}}}{L^{\frac{1}{2}} N^{\frac{1}{4}}}, \quad \mathrm{~s}_{\nabla}\left(D_{\nabla}^{*}\right)=\frac{3(v L)^{\frac{1}{2}}}{N^{\frac{1}{4}}}$

which provides a rule-of-thumb to chose the formation radius if $L, v$ and $N$ are known. The previous expression shows that both the minimal estimation error and the optimal formation radius decrease as the number of robots increases, however it is important to keep in mind that we must have $\left\|\mathbf{r}_{i}-\mathbf{r}_{j}\right\|>D_{\text {min }}$ for the previous equation to be realistic.

We now consider the effect on the Hessian estimation. Since even in this case the noise is additive in the estimate of the Hessian, it is straightforward to see that to the estimate $\widehat{\mathbf{H}}_{\sigma(\mathbf{c})}$ there is the additional term:

$$
\mathbf{\Psi}_{v}^{H}(D, \mathbf{c})=\frac{18}{N D^{4}} \sum_{i=1}^{N+2}\left(v_{i}-v_{c}\right)\left(\mathbf{r}_{i}-\mathbf{c}\right)\left(\mathbf{r}_{i}-\mathbf{c}\right)^{T}
$$

Such matrix has clearly zero mean, while the expected standard deviation in terms of the Frobenius norm is given by:

$$
\begin{aligned}
\mathbf{s}_{H}(D): & =\left(\mathbb{E}\left[\left\|\Psi_{v}^{H}(D, \mathbf{c})\right\|_{F}^{2}\right]\right)^{\frac{1}{2}} \\
& =\mathbb{E}\left[\operatorname{trace}\left(\Psi_{v}^{H}(D, \mathbf{c})\left(\Psi_{v}^{H}(D, \mathbf{c})\right)^{T}\right)\right] \\
& =\frac{18}{N D^{4}}\left(\mathbb{E}\left[\sum_{i=1}^{N+2} \sum_{j=1}^{N+2}\left(v_{i}-v_{c}\right)\left(v_{j}-v_{c}\right)\left(\widetilde{\mathbf{r}}_{i}^{T} \widetilde{\mathbf{r}}_{j}\right)^{2}\right]\right)^{\frac{1}{2}} \\
& =\frac{18}{N D^{4}}\left(\sum_{i=1}^{N+2} \mathbb{E}\left[v_{i}^{2}\right]\left\|\widetilde{\mathbf{r}}_{i}\right\|^{4}+\sum_{i=1}^{N+2} \sum_{j=1}^{N+2} \mathbb{E}\left[v_{c}^{2}\right]\left(\widetilde{\mathbf{r}}_{i}^{T} \widetilde{\mathbf{r}}_{j}\right)^{2}\right)^{\frac{1}{2}} \\
& =\frac{18 v}{N D^{2}}\left(N+2+\left(\frac{N}{3}+2\right)^{2}\right)^{\frac{1}{2}} \approx \frac{6 v}{D^{2}},
\end{aligned}
$$

where the last equation has been obtained via tedious but straightforward calculations which are reported in [18] in the interest of space, and the last approximation is obtained for $N \gg 1$. As compared to the effect of noise in the gradient estimation $\mathrm{s}_{\nabla}(D)$, the standard deviation $\mathrm{s}_{H}(D)$ does not decrease to zero as the number of robots increases, and it is also more sensitive to the formation radius since it is inversely proportional to the square of the radius. This last fact is to be expected since we are estimating second order derivatives via finite differences and therefore measurement noise is amplified more than when computing first derivatives.

Similar consideration can be derived for the Hessian in terms of finding the optimal radius $D_{H}^{*}$ to balance the approximation error and the noise error in the scenario $N \gg 1$ :

$\mathrm{s}_{H}\left(D_{\nabla}^{*}\right)=18 M D_{H}^{*} \Rightarrow D_{H}^{*}=\left(\frac{v}{3 M}\right)^{\frac{1}{3}}, \mathrm{~s}_{H}\left(D_{H}^{*}\right)=\frac{18(v M)^{\frac{1}{3}}}{3^{\frac{1}{3}}}$.

From a practical perspective, the optimal radius formation should be chosen to balance all approximation errors and noise effects in both the gradient and the Hessian. Since the noise error increases rapidly for decreasing formation radius a 


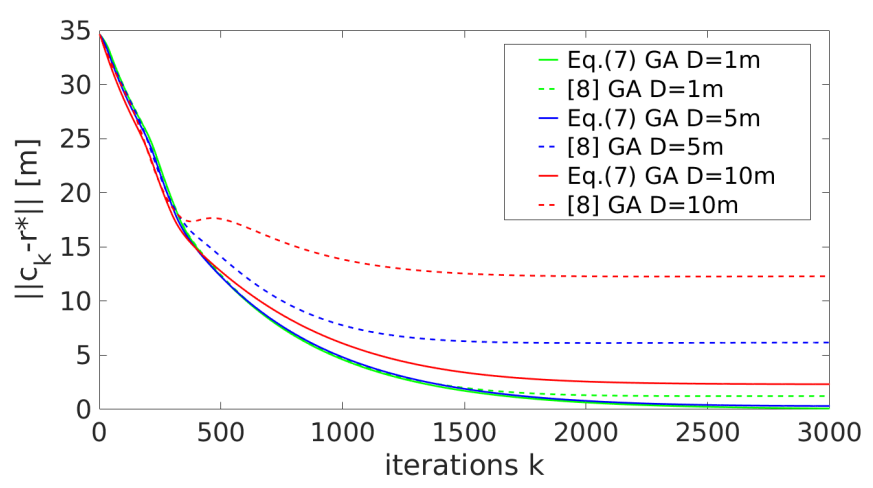

Fig. 3: Evolution of the distance between the center of the formation and the source location for the GA algorithm when the gradient is estimated via the least-squares method in [8] (dashed lines) and using our proposed approach (solid lines).

sensible choice for the radius is the largest between $D_{\nabla}^{*}$ and $D_{H}^{*}$.

\section{NUMERICAL SIMULATIONS}

In this section, we present numerical simulations to illustrate the performance of the proposed cooperative estimation algorithms applied to the source seeking problem. The signal strength considered is a 3-D Gaussian function whose surface sets are ellipsoids given by

$$
\sigma(\mathbf{r})=100 e^{-\mathbf{r}^{T} \mathbf{S r}},
$$

where $\mathbf{S}=10^{-4}\left[\begin{array}{ccc}100 & 1 & 1 \\ 1 & 1 & 0 \\ 1 & 0 & 10\end{array}\right]$. The maximum corresponding to the source is located at $\mathbf{r}^{*}=[0,0,0]^{T}$ denoted by the black $\times$.

We start by first comparing the quality of the proposed approximation for the gradient in a 3-D scenario with an alternative solution presented in [8], which is representative of many other algorithms based on least-squares solutions as in [7], [9]. Fig. 3 compares the results of the implementation of the GA algorithm using the gradient estimation proposed in Theorem 3 with respect to the gradient estimation proposed in [8]. Both algorithms were implemented using the same stepsize $\varepsilon=0.1$, the same formation of $N=8$ robots as defined in (6) and no noise was injected in order to evaluate only the approximation error. The evolution of the distance between the formation center and the source location, i.e., $\left\|\mathbf{c}_{k}-\mathbf{r}^{*}\right\|$, at each time step for three different values of the formation radius is shown. The geometrical properties of our proposed symmetric formation lead to a much better estimation of the gradient and thus the center of the formation is driven much closer to the proximity of the source location. It can be seen that the error of our proposed estimation strategy considerably outperforms the least-squares based method from [8]. Similar strategies presented in literature which do not exploit the geometric properties of the robots' formation exhibit analogous limitations.

In the next simulation we want to evaluate the benefit of using Hessian information to improve rate of convergence for source seeking applications, and to evaluate the impact of noise and approximation error. Fig. 4 shows a simulation

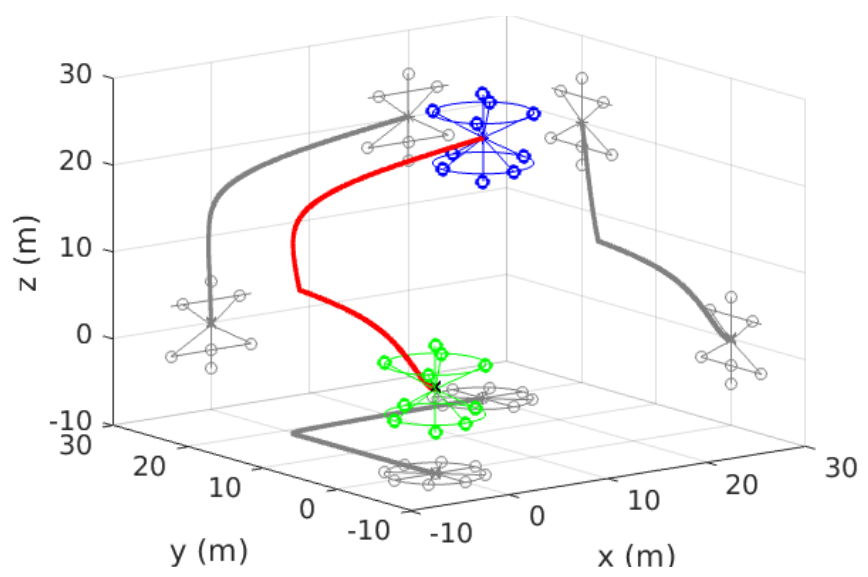

Fig. 4: 3D symmetric formation of 11 robots performing a source seeking mission based on the gradient and Hessian cooperative estimation of the signal at the formation center.

of $N=8$ robots uniformly spaced along a symmetric 3-D formation of radius $D=5 \mathrm{~m}$ as defined in (6) and three additional robots placed one at the center and two along of the $z$-axis as explained in Section IV-C, implementing the NRA defined in Section $\Pi$ with $\varepsilon=0.1$ and $\alpha=0.001$. The initial positions of the robots are represented by blue circles and the green ones denote the final positions. The red dots represent the trajectory of the formation center. The gradient and the Hessian matrix at the formation center are estimated at each time instant $k$ thanks to the computations (7), (10) and (13).

In order to evaluate the performance of our proposed strategy we also simulate two ideal algorithms implementing the GA and NRA source seeking defined in Section II assuming exact computation of the gradient and the Hessian at the center of the formation, thus providing a baseline for performance evaluation in a more realistic multi-robot scenario with noisy measurements. The evolution of these two algorithms is shown in Fig. 5 in black pointed and dashed lines, respectively. Fig. 5a also displays the evolution of the NRA algorithm implemented with three different values for the symmetric formation radius with $\varepsilon=0.1$ and $\alpha=0.001$ when the signal measurements are corrupted by white noise with $v=2$. For each iteration of the algorithm, the average over 100 trials of the distance between the center of the formation and the source is provided and the standard deviation is reported each 1000 iterations to make figures easier to read. As discussed in Section $\mathrm{V}$, these curves clearly show the trade-off between approximation error and noise error. If the formation radius is chosen too small $(D=1 \mathrm{~m})$, then the noise error dominates and the estimation of the Hessian is so poor that it is not even negative definite and therefore never used in the actual algorithm. As so, the NRA algorithm becomes a simple GA and no benefit in estimating the Hessian appears. If the formation radius is instead chosen too large $(D=10 \mathrm{~m})$, then the approximation error dominates and the center of the formation converges to a point which can be far from the true source. Differently, if the radius is properly chosen to balance both measurement noise error and approximation error, then 


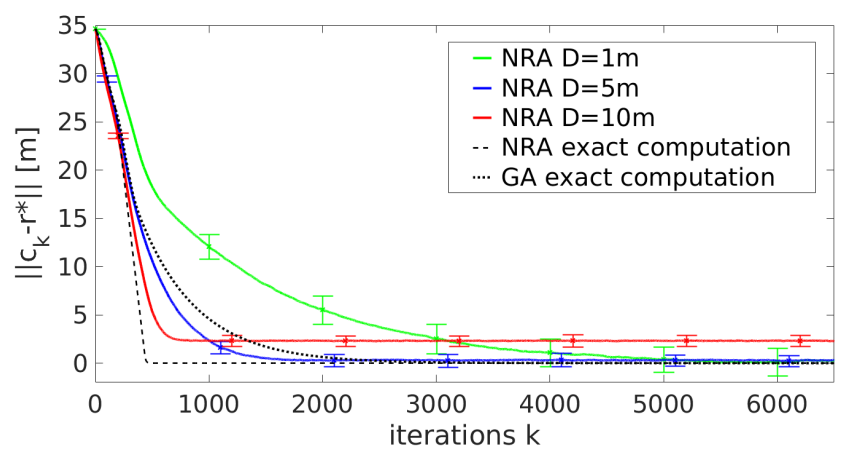

(a) Noisy measurements

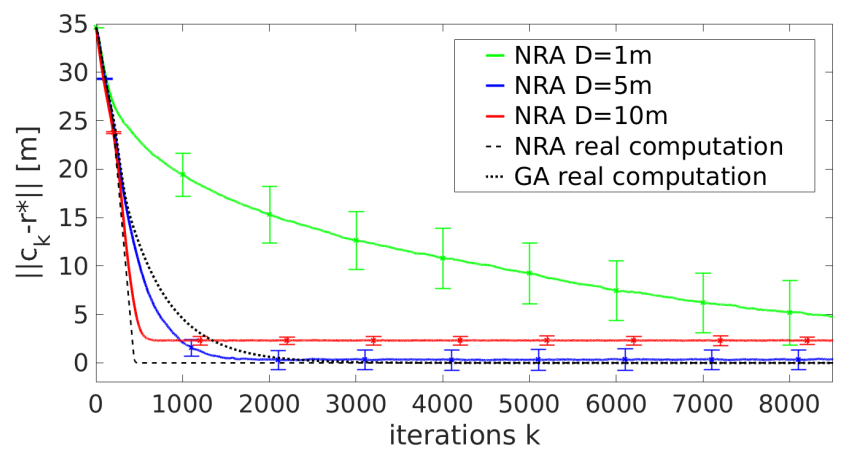

(b) Formation errors

Fig. 5: Mean and standard deviation over 100 trials of the distance between the center of the formation and the source location in presence of: (a) noisy measurements with $v=2$ and $(b)$ errors in robots' positions with $\delta=0.1 \mathrm{~m}$.

convergence behavior is very close to the ideal NRA with no noise and no approximation error.

With a view to study the robustness of our proposed estimation strategy with respect to formation errors, i.e., when the robots' positions do not exactly fit with the desired symmetric formation, we considered a perturbation vector $\boldsymbol{\delta}_{i}=\left[\delta_{x i}, \delta_{y i}, \delta_{z i}\right]^{T}$ where $\delta_{x i}, \delta_{y i}, \delta_{z i}, \sim \mathcal{N}\left(0, \delta^{2}\right)$ such that the perturbed position of each robot $i$ is defined as $\mathbf{r}_{i}^{p}=\mathbf{r}_{i}+\boldsymbol{\delta}_{i}$, where $\mathbf{r}_{i}$ is the desired robot's position in the symmetric formation. Fig. $5 \mathrm{~b}$ shows the average over 100 trials of the distance between the center of the formation and the source for each iteration of the NRA algorithm using the same 3-D 11robots formation as in the previous case, with three different values of the radius formation $D=1,5$, and $10 \mathrm{~m}$, considering now misalignment in the robots' positions with $\delta=0.1 \mathrm{~m}$. The standard deviation is reported each 1000 iterations. As shown in the figure, the gradient and Hessian estimation errors due to the perturbed robots' positions increase for smaller values of the formation radius. This behavior is similar to the noisy measurements case and again the formation radius plays an important role to balance approximation error and formation error. It is however important to remark that, even if our strategy strongly relies on the symmetric geometrical properties of the formation, the estimations computed in presence of errors in the robots' positions can be still exploited to locate the source and the estimation errors remain bounded.

\section{CONCLUSION}

This paper deals with the cooperative estimation of the gradient and Hessian matrix of a signal in the context of source seeking missions. We showed that the signal measurements collected by a planar circular formation of robots allow estimating both quantities at the center of the formation for the 2-D case. Moreover, another symmetric formation is proposed to deal with 3-D scenarios. In both cases, a limited number of robots and low computations are required to compute the estimates whose error is bounded and depends on the radius of the formation. Additionally, we mathematically proved that both the gradient and the Hessian can be exactly calculated for quadratic signals. Thanks to these results, the convergence rate of Newton-Raphson based source seeking algorithms is improved as shown in numerical simulations. A detailed analysis of the effect of noisy measurements in both the gradient and the Hessian estimations is provided. A further analysis of the effect of formation errors has been carried out in simulation, showing the robustness of our approach also with respect to errors in the robots' positions.

The design of an adaptive formation radius to increase the convergence rate of the Newton-Rhapson-based source seeking algorithm will be a key point in future research.

\section{ACKNOWLEDGMENT}

The work of L. Schenato was supported in part by the U. of Padova grant SID 2017 MAgIC.

\section{APPENDIX}

This appendix presents several relations and trigonometric properties used to prove the theorems and corollaries proposed in this paper. An important property of the symmetric formations proposed in this paper is that $\sum_{i=1}^{N} \cos \left(\frac{m 2 \pi}{N}\right)=$ $\sum_{i=1}^{N} \sin \left(\frac{m 2 \pi}{N}\right)=0$, for $N>m$. To prove this result, consider the well known geometric series property $\sum_{i=1}^{N} r^{i}=$ $\frac{r\left(1-r^{N}\right)}{1-r}, r \neq 1$. If we take $r=e^{\frac{m 2 \pi}{N} j}$ where $j^{2}=-1$ and $N>m$ to avoid the situation in which $r=1$, then

$$
\sum_{i=1}^{N} e^{\frac{m 2 \pi}{N} i j}=\frac{e^{\frac{m 2 \pi}{N} j}\left(1-e^{m 2 \pi j}\right)}{1-e^{\frac{m 2 \pi}{N} j}}=0
$$

since $e^{m 2 \pi j}=\cos (m 2 \pi)+j \sin (m 2 \pi)=1, \forall m$. Combining this result with trigonometric properties we obtain the following useful equations:

$$
\begin{aligned}
& c_{\phi}^{2}=\left(1+c_{(2 \phi)}\right) / 2, \quad c_{\phi}^{2} s_{\phi}=s_{\phi}-s_{\phi}^{3} \quad c_{\phi} s_{\phi}^{2}=c_{\phi}-c_{\phi}^{3}, \\
& c_{\phi}^{3}=\left(3 c_{\phi}+c_{(3 \phi)}\right) / 4, \quad c_{\phi}^{3} s_{\phi}=3 s_{(2 \phi)} / 8+\left(s_{(4 \phi)}-s_{(2 \phi)}\right) / 8, \\
& s_{\phi}^{3}=\left(3 s_{\phi}-s_{(3 \phi)}\right) / 4, \quad c_{\phi} s_{\phi}^{3}=3 s_{(2 \phi)} / 8-\left(s_{(4 \phi)}+s_{(2 \phi)}\right) / 8, \\
& c_{\phi}^{4}=3 / 8+c_{(2 \phi)} / 2+c_{(4 \phi)} / 8, \quad c_{\phi} s_{\phi}=s_{\phi} / 2, \\
& s_{\phi}^{4}=c_{\phi}^{4}-c_{(2 \phi)}, \quad c_{\phi}^{2} s_{\phi}^{2}=1 / 2+c_{(2 \phi)} / 2-c_{\phi}^{4} .
\end{aligned}
$$

\section{REFERENCES}

[1] P. Ogren, E. Fiorelli, and N. E. Leonard, "Cooperative control of mobile sensor networks: Adaptive gradient climbing in a distributed environment," IEEE Trans. on Aut. Cont., vol. 49, pp. 1292-1302, 2004.

[2] V. Kumar, D. Rus, and S. Singh, "Robot and sensor networks for first responders," IEEE Pervasive Computing, vol. 3, no. 4, pp. 24-33, 2004.

[3] L. Marques, U. Nunes, and A. de Almeida, "Particle swarm-based olfactory guided search," Auton. Robots, vol. 20, pp. 277-287, 2006. 
[4] W. Li, J. Farrell, S. Pang, and R. Arrieta, "Moth-inspired chemical plume tracing on an autonomous underwater vehicle," IEEE Trans. Robot., vol. 22, no. 2, pp. 292-307, 2006.

[5] B. Bayat, N. Crasta, A. Crespi, A. M. Pascoal, and A. Ijspeert, "Environmental monitoring using autonomous vehicles: a survey of recent searching techniques," Curr. Op. in Biotech., vol. 45, pp. 76-84, 2017.

[6] S. Z. Khong, Y. Tan, C. Manzie, and D. Nesić, "Multi-agent source seeking via discrete-time extremum seeking control," Automatica, vol. 50, pp. 2312-2320, 2014.

[7] J. Ryan and J. Speyer, "Peak-seeking control using gradient and hessian estimates," in Proc. of American Control Conference, 2010, pp. 611616.

[8] S. Zhu, D. Wang, and C. B. Low, "Cooperative control of multiple uavs for source seeking," J. Intell. Robot. Syst, vol. 70, pp. 293-301, 2013.

[9] S. Li, R. Kong, and Y. Guo, "Cooperative distributed source seeking by multiple robots: Algorithms and experiments," IEEE Trans. Mechatron. vol. 19, no. 6, pp. 1810-1820, 2014.

[10] W. Wu and F. Zhang, "Robust cooperative exploration with a switching strategy," IEEE Trans. on Robotics, vol. 28, no. 4, pp. 828-839, 2012.

[11] N. Leonard, D. Paley, F. Lekien, R. Sepulchre, D. Fratantoni, and R. Davis, "Collective motion, sensor networks, and ocean sampling," Proc. of the IEEE, vol. 95, no. 1, pp. 48-74, 2007.

[12] J. Cochran, A. Siranosian, N. Ghods, and M. Krstić, "3-D source seeking for underactuated vehicles without position measurement," IEEE Trans. on Robotics, vol. 25, no. 1, pp. 117-129, 2009.

[13] J. Lin, S. Song, K. You, and C. Wu, "3-D velocity regulation for nonholonomic source seeking without position measurement," IEEE Trans. on Control Sys. Tech., vol. 24, no. 2, pp. 711-718, 2016.

[14] R. Fabbiano, C. Canudas de Wit, and F. Garin, "Source localization by gradient estimation based on poisson integral," Automatica, vol. 50, no. 6, pp. $1715-1724,2014$.

[15] R. K. Williams and G. S. Sukhatme, "Probabilistic spatial mapping and curve tracking in distributed multi-agent systems," in Proc. of the IEEE Int. Conf. on Robotics and Automation, 2012, pp. 1125-1130.

[16] L. Briñón-Arranz, L. Schenato, and A. Seuret, "Distributed source seeking via a circular formation of agents under communication constraints," IEEE Trans. on Cont. of Network Sys., vol. 3, no. 2, pp. 104-115, 2016.

[17] L. Briñón-Arranz, A. Seuret, and C. Canudas-de-Wit, "Cooperative control design for time-varying formations of multi-agent systems," IEEE Trans. on Automatic Control, vol. 59, no. 8, pp. 2283-2288, 2014.

[18] L. Briñón-Arranz, A. Renzaglia, and L. Schenato, "Technical report on: Multi-robot symmetric formations for gradient and Hessian estimation with application to source seeking," https://hal.inria.fr/hal-01989573/ document 